\title{
A Piece of the Public Health Surveillance Puzzle: Social Contacts among School-Aged Children
}

\author{
Molly Leecaster*1, Warren Pettey ${ }^{1}$, Damon Toth ${ }^{1}$, Jeanette Rainey ${ }^{2}$, Amra Uzicanin $^{2}$ and \\ Matthew Samore ${ }^{1}$
}

${ }^{1}$ Internal Medicine, University of Utah, Salt Lake City, UT, USA; ${ }^{2}$ Centers for Disease Control and Prevention, Atlanta, GA, USA

\section{Objective}

To enhance public health surveillance and response for acute respiratory infectious diseases by understanding social contacts among school-aged children

\section{Introduction}

Timely and effective public health decision-making for control and prevention of acute respiratory infectious diseases relies on early disease detection, pathogen properties, and information on contact behavior affecting transmission. However, data on contact behavior are currently limited, and when available are commonly obtained from traditional self-reported contact surveys $[1,2]$. Information for contacts among school-aged children is especially limited, even though children frequently have higher attack rates than adults, and schoolrelated transmission is commonly predictive of subsequent community-wide outbreaks, especially for pandemic influenza.

Within this context, high-quality data are needed about social contacts. Precise contact estimates can be used in mathematical models to understand infectious disease transmission [3] and better target surveillance efforts. Here we report preliminary data from an ongoing $2-$ year study to collect social contact data on school-aged children and examine the transmission dynamics of an influenza pandemic.

\section{Methods}

Our aim is to capture mixing patterns and contact rates of schoolaged children in 24 schools and other non-school-related venues. We used a stratified design to ensure coverage of urban, suburban, and rural school districts, as well as climatically different areas (mountains and desert) in Utah. Elementary, middle, and high schools were chosen in each stratum. We defined a self-reported contact as anyone with whom the participant talked to face-to-face, played with, or touched. Contact logs collected subjective information (age, location, and duration) on self-reported contacts during a 2-day period. Objective contact data were collected by using proximity sensors [4] that recorded signals from other sensors within approximately $3-4$ feet.

Mixing patterns during school and non-school-related activities were summarized for participating school-aged children. We developed contact networks using proximity sensor data, providing visualizations of contact patterns as well as numeric contact measures. Contact networks were characterized with respect to degree distribution, and density. The degree for each person was calculated as the number of unique contacts. The density for a network was calculated as the number of observed contacts divided by the number of possible contacts.

\section{Results}

Two elementary schools, four summer camps, and one club participated in the study between May and August, 2012. Data were processed for the two schools and one camp. The mean degrees for the two schools were 28 and 29, with network sizes 109 and 129, respectively. The mean degree from camp was 43 , whose network size was 141 . The density of contacts was 0.26 and 0.22 for the schools and 0.31 for the camp. The density within classrooms at the two schools ranged from 0.78 to 0.98 . School-aged children typically underreported contacts using the contact log compared with objective proximity sensor data; this difference was statistically significant.

\section{Conclusions}

The variability in these and other contact network characteristics represent factors that could impact influenza transmission. Quantifying these factors improves our understanding of influenza transmission dynamics, which in turn can be used to adapt surveillance methods and control and prevention strategies. Almost all contact among students in our two elementary schools occurs within the classroom and the contact patterns differ by classroom, due to desk arrangement or other characteristics. Thus, during an elementary school outbreak it may be beneficial to focus on classroom-specific surveillance and control strategies.

The study is ongoing and we expect the variability in contact rates and mixing patterns will be even greater for middle and high schools where students switch classrooms and classmates each period. These schools could benefit from alternative surveillance and control strategies that account for the heightened overall mixing of the student body.

\section{Keywords}

children; respiratory infectious disease; social network; transmission model; proximity sensor

\section{Acknowledgments}

This study is funded by the Centers for Disease Control and Prevention 5U01CK000177.

\section{References}

1. Mossong, J, et al 2008 PLOS Medicine 5(3): 381-391.

2. Glass L and R Glass 2008 BMC Public Health 8(61).

3. Keeling M and K Eames 2005 J.R.Soc.Interface 2:295-307.

4. Salathé M, et al 2010 PNAS 1009094108.

*Molly Leecaster

E-mail: molly.leecaster@hsc.utah.edu 